Presented at the SPIE 33rd Annual International

BNL-42519 Technical Symposium on Optical \& Optoelectronic Applied Science and Engineering, San Diego, CA, August 6-11, 1989

\title{
SURFACE TOPOGRAPHY MEASUREMENTS OVER THE 1 METER TO 10 MICROMETER SPATIAL PERIOD BANDWIDTH*
}

\author{
Peter Z. Takacs, Karen Furenlid, Robert A. DeBiasse \\ Brookhaven National Laboratory, Instrumentation Division, Upton, NY 11973 \\ and \\ Eugene L. Church \\ USA ARDEC, SMCAR-CCAC-CCL-EM-65N, Dover, NJ 07801
}

September 1989

*This research was supported by the U. S. Department of Energy: Contract No. DE-AC-02-76CH00016. 


\section{SURFACE TOPOGRAPHY MEASUREMENTS OVER THE 1 METER TO 10 MICROMETER SPATIAL PERIOD BANDWIDTH*}

Peter Z. Takacs, Karen Furenlid, Robert A. DeBiasse

Brookhaven National Laboratory, Instrumentation Division, Upton, NY 11973 and

Eugene L. Church

USA ARDEC, SMCAR-CCAC-CCL-EM-65N, Dover, NJ 07801

September 1989

*This research was supported by the U. S. Department of Energy: Contract No. DE-AC-02-76CH00016. 


\section{DISCLAIMER}

This report was prepared as an account of work sponsored by an agency of the United States Government. Neither the United States Government nor any agency thereof, nor any of their employees, nor any of their contractors, subcontractors, or their employees, makes any warranty, express or implied, or assumes any legal liability or responsibility for the accuracy, completeness, or usefulness of any information, apparatus, product, or process disclosed, or represents that its use would not infringe privately owned rights. Reference herein to any specific commercial product, process, or service by trade name, trademark, manufacturer, or otherwise, does not necessarily constitute or imply its endorsement, recommendation, or favoring by the United States Government or any agency, contractor, or subcontractor thereof. The views and opinions of authors expressed herein do not necessarily state or reflect those of the United States Government or any agency, contractor or subcontractor thereof. 
Surface topography measurements over the 1 meter to 10 micrometer spatial period bandwidth*

Peter Z. Takacs, Karen Furenlid, Robert A. DeBiasse

Brookhaven National Laboratory, Upton, NY 11973

and

Eugene L. Church

USA ARDEC, SMCAR-CCAC-CCL-EM-65N, Dover, NJ 07801

\begin{abstract}
A recently-developed long-trace surface profiling instrument (LTP) is now in operation in our laboratory measuring surface profiles on grazing incidence aspheres and also conventional optical surfaces. The LTP characterizes surface height profiles in a non-contact manner over spatial periods ranging from 1 meter (the maximum scan length) to $2 \mathrm{~mm}$ (the Nyquist period for $1 \mathrm{~mm}$ sampling period) and complements the range of our WYKO NCP-1000 $2.5 \mathrm{X}$ surface roughness profiler $(5 \mathrm{~mm}$ to $9.8 \mu \mathrm{m}$ ). Using these two instruments, we can fully characterize both figure and finish of an optical surface in the same way that we normally characterize surface finish, e.g., by means of the power spectral density function in the spatial frequency domain. A great deal of information about the distribution of figure errors over various spatial frequency ranges is available from this data, which is useful for process control and predicting performance at the desired wavelength and incidence angle. In addition, the LTP is able to measure the absolute radius of curvature on long-radius optics with high precision and accuracy. Angular errors in the optical head are measured in real time by an electronic autocollimator as the head traverses the linear air bearing slide. Measurements of kilometer radius optics can be made very quickly and the data analyzed in a format that is very easy to understand.
\end{abstract}

\title{
1. INTRODUCTION
}

The quality of aspheric optics has been of great concern to users of synchrotron radiation (SR) especially since the proliferation of dedicated storage ring sources in the early 1980's. The difficulties in manufacturing and measuring the figure and finish of large cylindrical aspheres used to collect and focus soft $x$-rays from SR and also from astronomical sources are well documented. ${ }^{14}$ The procurement of suitable grazing incidence optical components from small and large shops has been hindered by the lack of suitable standards and testing methods for these unconventional surfaces. Conventional full-aperture interferometric figure testing is totally inadequate to handle off-axis cylinders, toroids, and ellipso: .; found in highly-decentered grazing incidence SR beamlines. Initial attempts by NSLS users to procure suitable componesis were not successful: the poor quality of the optical components compromised the quality of the high-brightness sources and prevented the optical systems from reaching their full design potential.

\section{SURFACE FINISH}

In order to investigate problems related to the manufacture of $x$-ray optics, a research program was initiated to develop metrology techniques suitable for the characterization of full-size SR mirrors. Our initial goal was to improve the finish of grazing incidence optics to reduce the amount of light scattered out of the specular direction at short $x$-ray and EUV wavelengths. We were fortunate in that, at that time, a commercial micro-interferometer system was developed which was ideally suited for making surface roughness measurements on full-size optical components over the spatial bandwidth region of interest to us. ${ }^{5}$ A significant effort was made to understand the performance characteristics of the WYKO optical profiler instrument and to develop a practical measurement technique that could be used as the basis for a surface roughness specification. The success of our program is evidenced by a significant improvement in the quality of optical surfaces now delivered for use at the $\mathrm{NSLS}^{6}$ and by a recent proposal to develop an ISO surface roughness standard based on the power spectrum of the roughness. ${ }^{7}$ 


\section{SURFACE FIGURE}

Although we were successful in employing the microinterferometer in measuring the roughness of smooth surfaces, there still remained the problem of measuring the overall shape, or figure, of cylindrical aspheres. In order to utilize the small source size (vertical height < 150 micrometers) and take advantage of the inherent high brightiess of the NSLS sources, the image formation properties of the mirrors needed improvement. As no commercial instrumentation was available to make figure measurements on cylindrical aspheres, we embarked on a development program to produce an instrument that would allow us to measure the figure on cylinders up to one meter in length. Since we already had a quick and reliable method for measuring microroughness with scale lengths ranging from several micrometers up to several millimeters, we decided to build a separate instrument to cover the complementary spatial period range, from one millimeter up to 1 meter, rather than try to build a new instrument that encompassed the entire spatial frequency range. Our approach to cylindrical figure measurement was to employ a pencil beam interferometer" to make profile measurements down the lengih of the mirror in a non-contact manner and use the same techniques to analyze the figure data as we use to analyze the finish data."

The power spectral density of the surface profile has proved to be a very useful analytic tool for understanding the nature of polished surfaces and for predicting the performance of grazing incidence optics. The Fourier spectrum of the axial profile of cylindrical mirrors over the composite spatial frequency range of the two complementary profiling instruments can be used to predict the complete imaging and scattering performance of the component ${ }^{10}$ and can be used as a specinication to the manufacturer for the production of the desircd surface. It serves as the point of commonality between the functional performance of grazing incidence systems at $x$-ray wavelength and the metrology made at visible wavelengths in the laboratory. The power spectral density approach to surface specification is not widely used, mainly because it is an unfamiliar concept to most traditional fabricators and because commercial interferometer analysis software does not support the calculation. One finds it used in specialized projects involving the development of sophisticated optical systems, such as $x$-ray telescope projects, ${ }^{11}$ but with the new measurement techniques now available, it should become a more common specification technique.

\section{SURFACE FIGURE MEASUREMENT}

Measurement of microroughness over the $5 \mathrm{~mm}$ to $10 \mu \mathrm{m}$ spatial period bandwidth has been adequately covered in numerous earlier publications. ${ }^{69.12-15}$ The remainder of this paper will be concerned with extending the range of surface profile measurements to 1 meter distances. The instrument that we have developed in our laboratory to achieve that goal is the Long Trace Profiler (LTP). This instrument has been described in detail elsewhere, ${ }^{16,17}$ so only a brief discussion of its operation is in order now. The LTP consists of an optical head mounted on an air bearing slide driven by a stepping motor lead screw drive with a free travel range of almost one meter. The optical head consists of a HeNe laser source, beamsplitting optics to produce two collinear but otherwise unaltered probe beams, and a lens and linear array detector assembly to record the interference pattera produced by the two beams after reflection from the test surface. Data collection and analysis are controlled by an HP320 computer system.

The LTP is a slope measuring interferometer. The mcasured parameter is the local axial surface slope between the two adjacent regions of the surface illuminated by the probe beams. The optical head is stepped along the surface in increments of one laser beam diameter $(1 \mathrm{~mm})$, and the measured slope values form the pixel function, which is surface slope in units of detector pixel number. The pixel function is converted into true slope by application of the calibration factor, which is 10.1 micrnradians per pixel. The slope function is then converted into height by a Fourier filtering tcchnique, ${ }^{817}$ and the power spectral density function can be generated through further processing.

An important design criterion of the LTP is that it be able to measure absolute radius of curvature. This is accomplished by monitoring the pitch angle of the optical head in real time as it moves along the air bearing rail. We use a sensitive electronic autocollimator to measure the change in angle of the optical head as a function of position along the rail. By subtracting the optical head pitch angle from the raw slope function, we cficctively remove all slope errors introduced 
by static and dynamic deflection of the air bearing beam. We do not need to rely on a precisely-calibrated special reference flat or straightedge in order to make absolute surface radius measurements: the nature of the LTP system is self-calibrating.

Two major sources of systematic error in LTP measurements are fluctuations in the local thermal environment and stability of the laser pointing direction. We are investigating the directional stability of several laser sources, including HeNe and diode lasers, to determine those which are best suited for the LTP. Noise in the laser beam pointing direction in the order of fractions of a microradian translates directly into apparent surface slope errors. The local thermai environment in our laboratory is not well suited for sub-microradian precision angle measurement: the room temperature usuaily fluctuates several degrees Fahrenheit three times an hour, especially in summer. A shroud over the instrument reduces the thermal fluctuations by about an order of nagnitude, but thermal equilibrium is never achieved. The HeNe laser is also a significant heat source within the local environment. The effect of slow thermal drift on the measurement can be reduced by speeding up the data acquisition process. Our present system is rather slow, taking 3 to 4 seconds to acquire a data point with a noise level of about 500 nanoradians per point. Our goal is to reduce the acquisition time to less than one second per point by taking data on the fly, rather than starting and stopping the translation slide for each point as is done now.

\section{TYPICAL MEASUREMENTS}

A large number of mirrors have been measured on the LTP over the past year. Most have been cylindrical aspheres, either in the shape of a partial right cylinder with a minor radius on the order of tens of millimeters (see Fig. 1), or in the shape of a toroid with a major radius on the order of kilometers or hundreds of meters. Some cylinders have been designed to be bent in the axial direction to approximate a toroid. The LTP has proven to be an extremely useful device for analyzing the performance of mirror bending mechanisms, allowing one to compare the actual shape of the bent mirror to the predicted shape as a function of applied load. Most cylinder mirrors that have been measured on the LTP suffer from common manufacturing defects. It is a useful exercise to review these problems in order to make them known to potential users of cylindrical aspheres and to feed back the metrology information to the manufacturer. The goal of this exercise is to stimulate improved production techniques for aspheric optics.

A schematic diagram of a typical grazing incidence cylinder mirror is shown in Fig. 1. Most cylinders in use at the NSLS are 60 to $70 \mathrm{~cm}$ in length. Most suffer from figure errors in common with the following example. The raw slope function measured on a diamond-machined and polished single crystal silicon mirror is shown in Fig. 2. Several features of interest are evident in this plot. The overall tilt of the curve from upper left to lower right indicates that this surface has a large non-zero curvature. (The cerivative of an ideal surface with curvature is a sloping straight line.) Something is wrong at each end of the mirror because the slope function departs from the average tilt and becomes steeper at each end. And most obvious is a uniform periodicity in the center segment with an amplitude of several microradians and a period of exactly 20 millimeters.

The surface height profile computed from the raw slope function is shown in Fig. 3. One can see immediately that the non-zero slope is the result of a large convex curvature in the surface with a sag of about $20 \mu \mathrm{m}$. Such a surface will not function well as a focusing element! Our analysis software allows us to remove the tilt from the slope function over the central region of the surface, which is equivaient to removing the curvature from the height profile. If we perform these steps and calculate the height from the detrended slope function, we see the residual surface profile in Fig. 4. This curve is the departure of the surface from the radius best-fit to the central $500 \mathrm{~mm}$ of the surface. One notes immediately that the steep slope regions near each end are a result of edge roll-off in the finished surface. Presumably the quality of the diamond-machined surface was compromised by the polishing techniques as evidenced in the edge roll-off region and in the overall convex curvature aiong the axis. Convex curvature and edge roll-off are common problems seen in most cylindrical surfaces in varying degrees. This is a rather extreme example of overall curvature but is a typical example of edge roll-off. The most unusual aspect of this surface does not show up well in the height profiles, except at higher magnification of the vertical scale, but is readily apparent in the slope function plots, that being the $20 \mathrm{~mm}$ periodicity in the central region of the surface. If we compute the power spectrum of the residual height profile of Fig. 4 and plot 
the result in Fig. 5, we can see the signature of the $20 \mathrm{~mm}$ periodicity standing out from the continuum background as a sharp spectral line. The area under the spectral line implies that the surface has an RMS roughness of $22 \AA$ at the $20 \mathrm{~mm}$ period, which is equivalent to a $20 \mathrm{~mm}$ period sinusoid with a peak-to-valley amplitude of $63 \AA$ extending over the length of the surface. The maximum slope error produced by this idealized sinusoid is $\pm 1 \mu \mathrm{rad}$, or about 0.2 arcseconds. This happens to be well within the specifications for this mirror surface, which allow several areseconds of error at these spatial frequencies. If the $20 \mathrm{~mm}$ periodicity were the only error source on this mirror, it would be acceptable. However, the $20 \mu \mathrm{m}$ convex sag renders the mirror unusable, even though it is intended to be used in a bending apparatus. The bender is designed to produce a concave sag of about $17 \mu \mathrm{m}$. If this mirror were installed, the bender would first have to unbend the $20 \mu \mathrm{m}$ convex curvature, which would require large forces outside the desired range of operation. This mirror was returned to the manufacturer for additional figuring.

Also shown in Fig. 5 at the higher spatial frequencies (greater than $2 \times 10^{-1}$ ) is the power spectrum of the silicon mirror surface measured with a WYKO NCP 1000 Digital Optical Profiler with a $2.5 \mathrm{X}$ objective. This is a result our standard measurement method and analysis technique to quantify the roughness characteristics of surfaces over the $5 \mathrm{~mm}$ to $10 \mu \mathrm{m}$ spatial period range. The WYKO-range spectrum is an average over several spectra calculated from profile measurements made at random locations along the length of the cylindrical surface. The most outstanding feature of the composite spectrum is the smooth transition between the two measurement techniques: the magnitude of the two spectra in the region of overlap is the same, although the profiles were measured by two completely different techniques, and the slope of the composite spectrum is constant over an enormous range of spatial frequencies, corresponding to periods from $100 \mathrm{~mm}$ down to $10 \mu \mathrm{m}$. The composite spectrum has a $1 / \mathrm{P}^{\mathrm{P}}$ power law shape with a spectral index of about $\mathrm{n}=-1.33$.

The surface spectrum can be used to compute the RMS roughness statistics of the surface by integrating the area under the curve over selected bandwidth regions. If we integrate over the WYKO region from $5 \mathrm{~mm}$ down to $10 \mu \mathrm{m}$, we get an RMS roughness value of $10.95 \AA$, which is a reasonably good finish for this type of mirror. The corresponding RMS roughness number for the LTP region over the $100 \mathrm{~mm}$ to $4 \mathrm{~mm}$ bandwidth is $57.5 \AA$, which includes the $22 \AA$ contribution from the spectral line at $20 \mathrm{~mm}$. The high frequency cutoff, e.g. Nyquist frequency, for the LTP data occurs at the $4 \mathrm{~mm}$ period because the sampling interval in this data set is $2 \mathrm{~mm}$ per data point. The large amplitude of the LTP spectrum at the longest spatial periods, between $500 \mathrm{~mm}$ and $100 \mathrm{~mm}$, results in an RMS roughness value of 934 $\AA$. The source of this large low frequency component is readily apparent in the residual height profile as the height difference between the left and right halves of the mirror. By eye, the peak-to-valley height difference appears to be somewhat less than $1 \mu \mathrm{m}$, which is in agreement with the computed RMS value of nearly $0.1 \mu \mathrm{m}$. Surface profile errors in this spatial period range are usually classified as figure errors and are usually expressed in units of waves or wavelengths of light. But there is usually some ambiguity in the meaning expressed in those terms as to whether the error refers to wavefront error or actual surface error. With the RMS error expressed in real distance units ( $\mu \mathrm{m}$, nm, or Ångstroms), there is no ambiguity in the meaning of residual surface height error.

A second example of an LTP measurement on another type of cylindrical asphere, a $300 \mathrm{~mm}$ long fused silica toroid, is shown in Fig. 6. The design parameters of this mirror are a major radius of 1500 meters and a minor radius of $13.0 \mathrm{~cm}$. As is typical with most cylindrical aspheres, the raw slope function is comprised of three distinct regions: a uniform-slope central zone and irregular zones at each end. The height profile computed from the slope function is shown in Fig. 7 and the residual height profile, after removal of the central radius of curvature, is shown in Fig. 8. The edge roll-off of several micrometers is clearly visible in the residual height profile and is typical of most long, narrow cylinder mirrors.

This mirror is unusual in that the manufacturer supplied us with his own metrology data which allowed us to confirm the validity of our absolute curvature measurement. The manufacturer measured the sag along the length of the toroid with a $10^{\circ}$ bar spherometer. His measured sag was 250 microinches $(6.35 \mu \mathrm{m})$ with an uncertainty in the range of $10-20$ microinches. The computed radius of curvature for this sag value is $1270 \mathrm{~m}$. If we make the same measurement using the height profile in Fig. 7, our measured sag is $6.47 \mu \mathrm{m}$ (254.7 microinch), which results in a major radius of $1246 \mathrm{~m}$. The sag and radius values measured by the two different techniques are in excellent agreement. 
Unfortunately, one measurement of the sag along a fixed baseline does not tell the whole story of this mirror. Notice from Fig. 7 that the bar spherometer measurement is made with the contact points in the roll-off regions at each end of the mirror. If we shorten our baseline to $200 \mathrm{~mm}$ instead of $254 \mathrm{~mm}$ to exclude the edge roll-off regions, and then measure the sag, we measure $5.04 \mu \mathrm{m}$ of sag and compute a major radius of $992 \mathrm{~m}$, which is significantly different from the $1246 \mathrm{~m}$ radius computed nver the larger baseline and is not even close to the $1500 \mathrm{~m}$ design parameter. The ability to measure and see the entire axial profile of this mirror has allowed us to compare and confirm our metrology techniques against an independent method and has allowed us to identify a potential source of systematic error in a common shop metrology technique.

The LTP offers a rapid and accurate means to measure the performance of mirror bending apparats. A common technique for producing axial curvature in a cylindrical or plane surface is to bend it with appropriately-placed force couples near each end of the mirror. Often the performance of the bending mechanism does not follow its design criteria and a period of debugging and modification is necessary to understand the behavior of the mechanism. The LTP allows one to assess the performance of the bending system off-line in a controlled laboratory environment. Examples of profile measurements on mirrors installed in two different bending mechanisms are shown in Figs. 9 and 10. Measurements on a thin fused silica cylinder bent into the shape of a toroid are shown in Fig. 9. The thin cross section (about $8 \mathrm{~mm}$ thick) is easily bent into the 100-200 meter radius range. The tolal sag over the $500 \mathrm{~mm}$ clear aperture is over $300 \mu \mathrm{m}$ for the $R=100 \mathrm{~m}$ case. Measurements over this sag depth range are difficult lor most other optical profiling systems. The limitation on the measurement range of the LTP is not one of total sag, but rather depends on the maximum slope of the surface. In the present configuration the aperture of the Fourier transform lens limits the maximum surface slope to be \pm 5 mrads. The above example bas a maximum slope of only \pm 2.5 mrads. The last example in Fig. 10 is for a single-crystal silicon mirror in a different bending mechanism. The mirror is a sister mirror to the one featured in Fig. 4 but does not exhibit the $20 \mathrm{~mm}$ periodicity error. It is, however, slightly more irregular than the first one. After a period of debugging problems with the bending mechanism, the mirror was successfully bent into the desired radii in the range from $4.9 \mathrm{~km}$ to $12.2 \mathrm{~km}$. This mirror is designed for use at extreme grazing incidence angles to focus kilovolt energy $x$-rays. The curvature on the silicen mirror is much shallower than the other mirror which is designed to focus much lower energy EUV photons at a shorter focal distance.

\section{CONCLUSION}

The measurement of the surface quality of large cylindrical aspheres is now a routine procedure in our laboratory. Profile measurements over the length of the mirror allow one to quickly and easily characterize the quality of the surface. The Long Trace Profiler allows us to measure the figure quality of large mirrors, and the WYKO profiler allows us to measure the finish. We have demonstrated that, at least in the case of the silicon mirror surface, the power spectral density function follows a power law curve over a wide range of spatial frequencies. Further work is necessary to characterize the spectra of other surfaces over the LTP range. We have also shown that certain manufacturing errors are common to cylindrical aspheres and that we have the metrology capability to provide the necessary feedback to the manufacturers so that they might improve their production techniques.

\section{REFERENCES}

1. Peter Z. Takacs, "Metrology of Reflection Optics for Synchrotron Radiation," Nucl. Instnum. \& Meths., A246, $227-241$ (1986).

2. R. H. Price, "X-ray Microscopy Using Grazing Incidence Reflection Optics," Low Energy X-ray Diagnostics, D. T. Attwood and B. L. Henke, eds., AIP Conference Proc. 75, 189 (1981).

3. G. Makosch and B. Solf, "Surface profiling by Electro-Optical Phase Measurements," High Resolution Soft Xray Oprics, Proc. SPIE 316,42 (1981). 
4. A. Franks, "The Metrology of X-ray Optical Components," Low Energy X-ray Diagnostics, David T. Attwood and Burton L. Henke, eds., AIP Conf. Proc. 75 , 179-188 (1981).

5. B. Bhushan, J. C. Wyant and C. L. Koliopoulos, "Measurement of Surface Topography of Magnetic Tapes by Mirau Interferometry," Appl. Opc. 24, 1489 (1985).

6. P. Z. Takacs, "Understanding the Performance of X-ray Mirrors," Synchrotron Radiation in Structural Biology, R. Sweet and A. Woodhead, eds., Plenum Press, New York, 1989.

7. D. J. Janeczko, "Power Spectrum Standard for Surface Roughness: Part I," Proc. SPIE 1165 (1989), in press.

8. K. von Bieren, "Interferometry of Wavefronts Reflected Off Conical Surfaces," Appl. Opt. 22, 2109 (1983).

9. E. L. Church and P. Z. Takacs, "Use of an Optical-profiling Instrument for the Measurement of the Figure and Finish of Optical-quality Surfaces," WEAR 109, 241-157 (1986).

10. E. L. Church and P. Z. Takacs, "Prediction of Mirror Performance from Laboratory Measurements," $X$ ray/EUV Optics for Astronomy and Microscopy, R. B. Hoover, ed., Proc SPIE 1160, 323-336 (1989).

11. Paul Glenn, "Space Telescope Performance Prediction Using the OSAC Code," Lange Optics Technology, Proc. SPIE 571, 164-179 (1985).

12. E. L. Church and P. Z. Takacs, "Survey of the Finish Characteristics of Machined Optical Surfaces," Opt. Eng. 24. 396 (1985).

13. E. L. Church and P. Z. Takaes, "Statistical and Signal Processing Concepts in Surface Metrology," Proc. SPIE 645, 107 (1986).

14. E. L. Church and P. Z. Takacs, "Instrumental Effects in Surface Finish Measurement," Surface Measurement and Characterization, J. M. Benneth, ed., Proc. SPIE 1009, 46-55 (1989).

15. E. L. Church, T. V. Vorberger and J. C. Wyant, "Direct Comparison of Mechanical and Optical Measurements of the Finish of Precision Machined Optical Surfaces," Opt. Eng. 24, 388 (1985).

16. Peter Z. Takacs, Shi-nan Qian and Jeffrey Colbert, "Design of a Long-Trace Surface Profiler," MetrologyFigure and Finish, Bruce Truax, ed., Proc. SPIE 749, 59-64 (1987).

17. P. Z. Takacs, S.-C. K. Feng, E. L. Church, S.-N. Qian, and W.-M. Liu, "Long Trace Profile Measurements on Cylindrical Aspheres," Advances in Fabrication and Metrology for Optics and Lange Optics, Jones B. Arnold and Robert E. Parks, eds., Proc. SPIE 966, 354-364 (1989). 


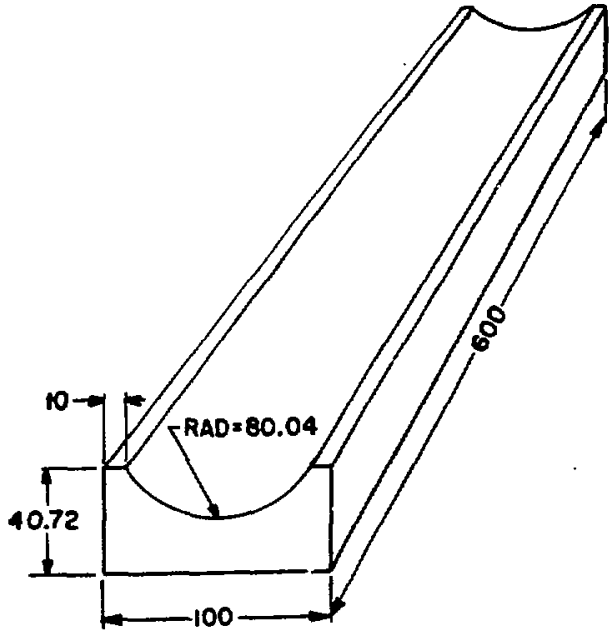

Fig. 1 Schematic of a typical $x$-ray cylinder mirror used to reflect synchrotron radiation at a grazing incidence angle of 3 mrads. Dimensions are in millimeters.

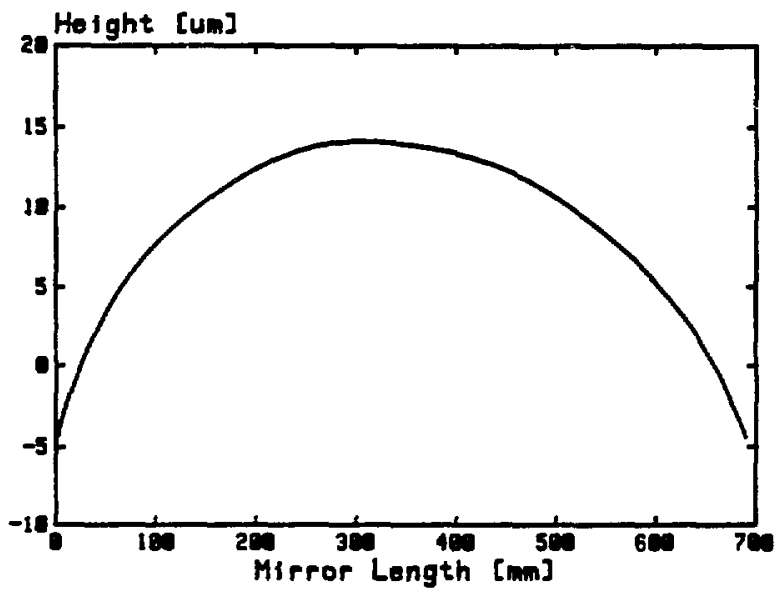

Fig. 3 Axial height profile of the single-crystal silicon mirror computed directly from the slope function in Fig. 2. The convex curvature with a sag of about $-20 \mu \mathrm{m}$ would require application of excessive force in the mirror bender to produce a concave curvature with the desired $+12 \mu \mathrm{m}$ sag.

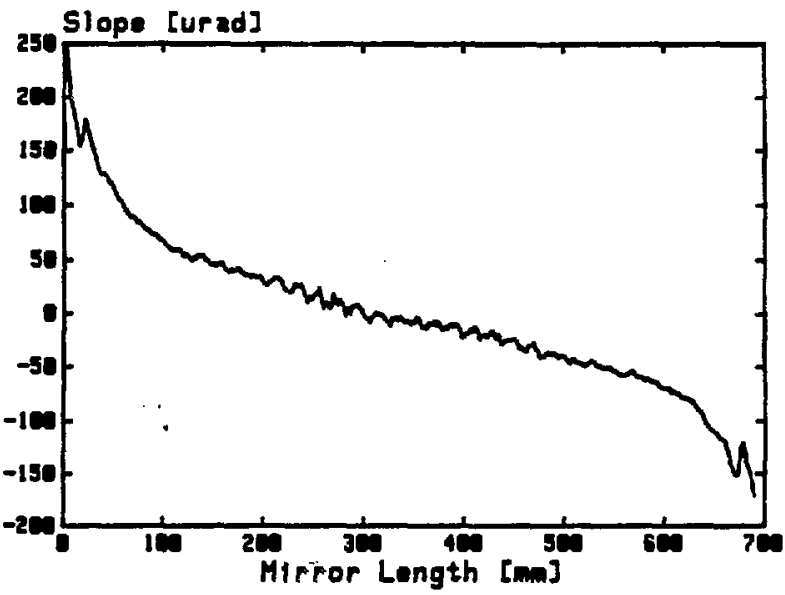

Fig. 2 Raw slope function for a $700 \mathrm{~mm}$ long singlecrystal cylinder mirror measured by the LTP. Vertical axis units are in microradians. The $20 \mathrm{~mm}$ periodicity in the surface is clearly visible.

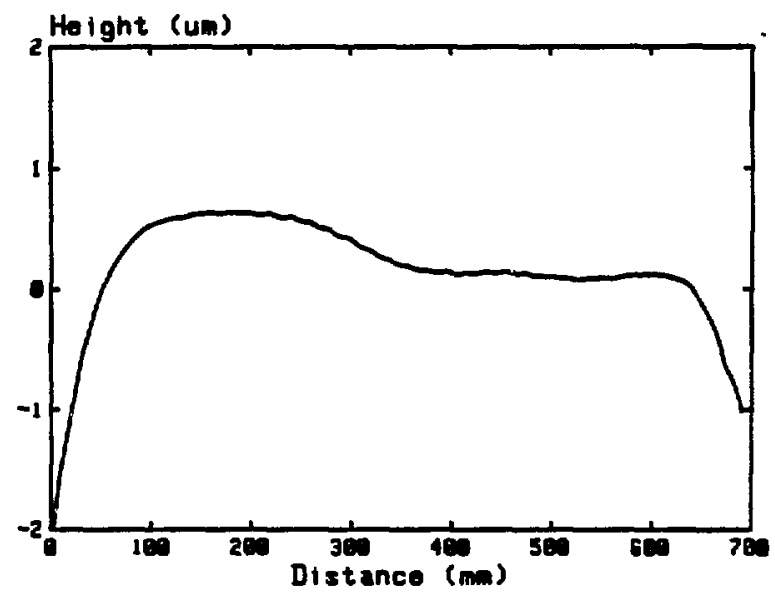

Fig. 4. Detrended axial height profile: average radius of curvature of center section of Fig. 3 has been subtracted. Edge roll-off is clearly visible in the residual profile. The $20 \mathrm{~mm}$ periodicity is barely visible at this vertical scale. 


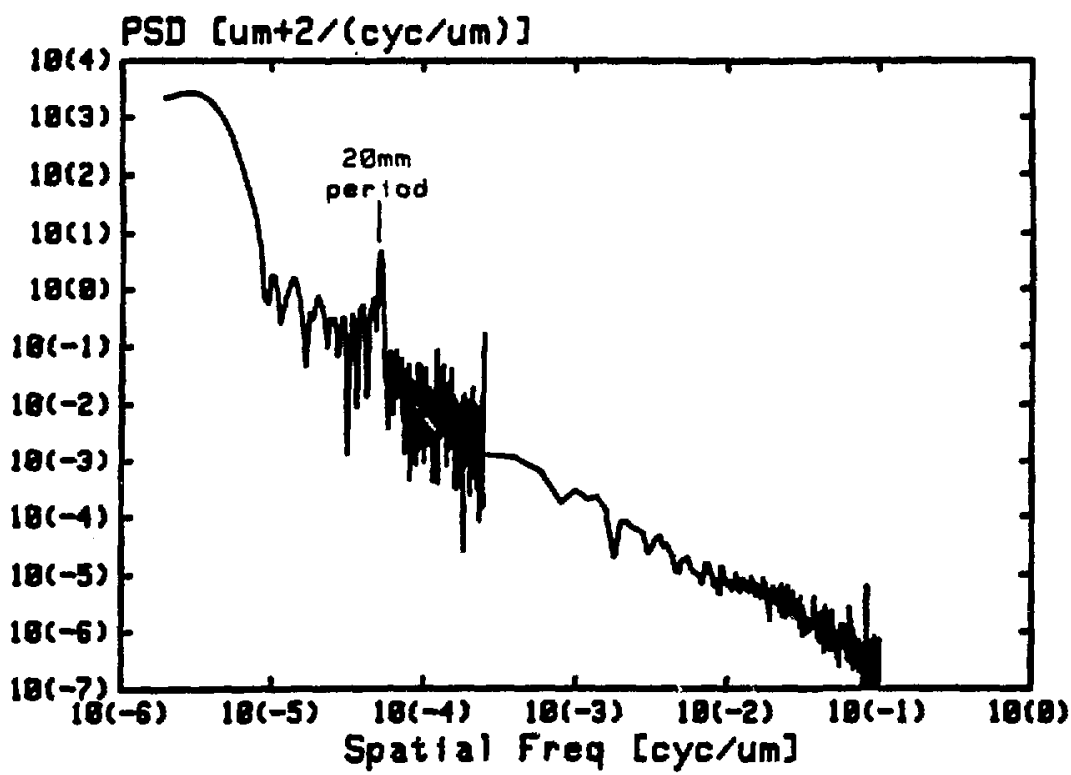

Fig. 5 Composite power spectrum of surface roughness for the silicon mirror. Lower right curve is an average from several WYKO profiler measurements, covering the spatial frequency range corresponding to pericds between $5 \mathrm{~mm}$ and $10 \mu \mathrm{m}$. Upper left curve is from the central $500 \mathrm{~mm}$ of the Fig. 4 height profile and covers spatial periods between $500 \mathrm{~mm}$ and $4 \mathrm{~mm}$. The composite spectrum follows a power law shape with spectral index $\mathrm{n}=-1.33$ over the $100 \mathrm{~mm}$ to $10 \mu \mathrm{m}$ period range. The $20 \mathrm{~mm}$ periodicity stands out clearly above the underlying continuum and is equivalent to a $63 \AA \mathrm{P}-\mathrm{V}$ sinusoid on the surface. The large increase in PSD at the longest spatial periods arises from the step height discontinuity between the two halves of the mirror.

Slope [urad]

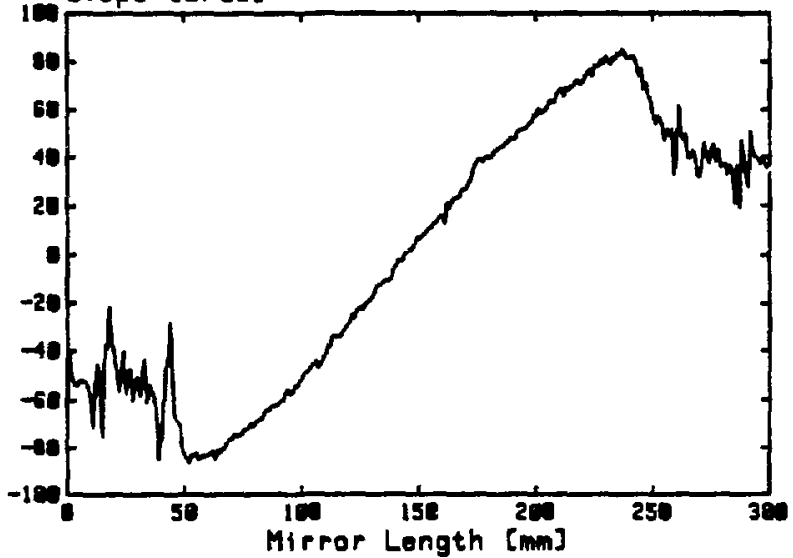

Fig. 6 Raw slope function for a fused silica toroidal mirror $300 \mathrm{~mm}$ long. Irregular regions at each end indicate problems with the polishing method.

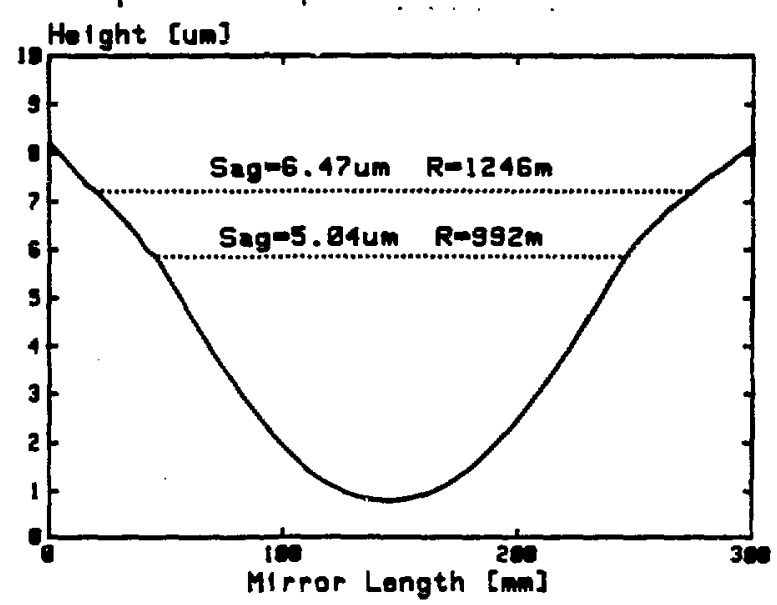

Fig. 7 Height profile computed from the Fig. 6 data. Design radius was 1500 meters. Dotted lines indicate sags and radii computed over two baselines. Upper line corresponds to manufacturer's measurement with a $10^{n}$ ball spherometer, which falls in the roll-off region; lower line excludes the edge roll-off. Computed radii are significantly different between the two measurements. 


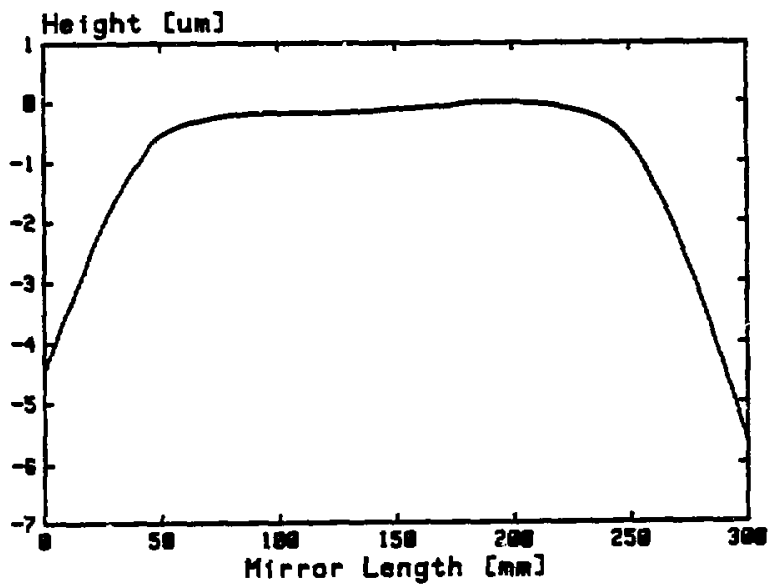

Fig. 8 Detrended height profile of the fused silica toroid with the 992 meter radius subtracted. Edge roll-off is quite severe.

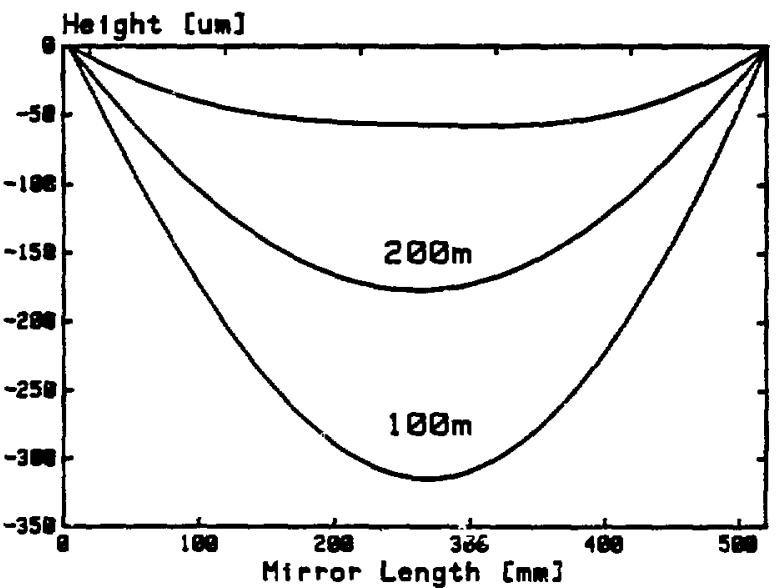

Fig. 9 Profiles of a thin fused silica cylinder bent into short radius toroidal shapes in a bending mechanism. The large sag illustrates the large depth of field capability of the LTP. Upper curve with small applied load has a non-circular shape, indicate a problem with the bending apparatus.

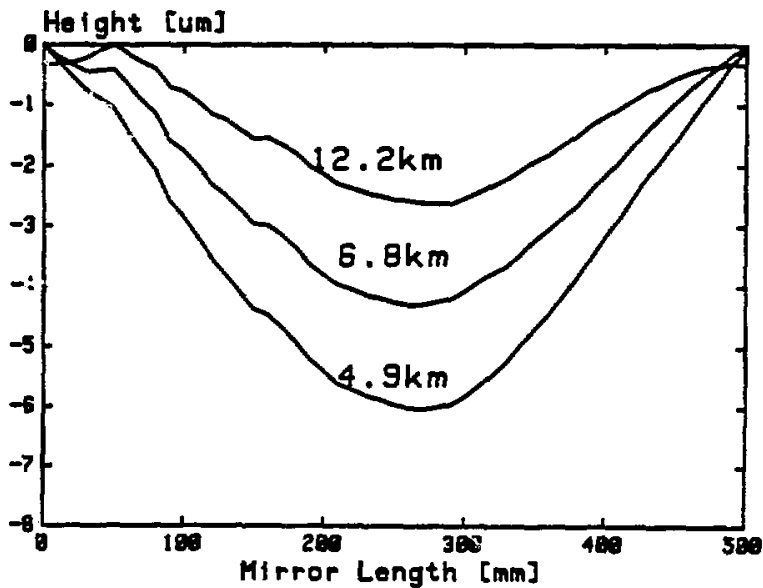

Fig. 10 Mirror bender measurements on a singlecrystal silicon mirror similar to the one in the above discussion. The bending mechanism needs only produce a small amount of curvature to achieve vertical focusing at the $3 \mathrm{mrad}$ grazing incidence angle. Accurate absolute curvature measurements are possible because of real-time angular measurement head error correction from an electronic autocollimator. 